\title{
Effective Connectivity of the Fronto-parietal Network during Attentional Control
}

\author{
Liang Wang ${ }^{1 *}$, Xun Liu ${ }^{1 *}$, Kevin G. Guise ${ }^{1}$, Robert T. Knight ${ }^{2}$, \\ Jamshid Ghajar ${ }^{3,4}$, and Jin Fan ${ }^{1}$
}

\begin{abstract}
The ACC, the dorsolateral prefrontal cortex (DLPFC), and the parietal cortex near/along the intraparietal sulcus (IPS) are members of a network subserving attentional control. Our recent study revealed that these regions participate in both response anticipation and conflict processing. However, little is known about the relative contribution of these regions in attentional control and how the dynamic interactions among these regions are modulated by detection of predicted versus unpredicted targets and conflict processing. Here, we examined effective connectivity using dynamic causal modeling among these three regions during a flanker task with or without a target onset cue. We compared
\end{abstract}

\section{INTRODUCTION}

The fronto-parietal network, including the ACC, the dorsolateral prefrontal cortex (DLPFC), and the cortex along the intraparietal sulcus (IPS), has been consistently shown to be recruited by tasks that involve top-down attentional control processes during processing of conflict between alternative responses and during bottom-up stimulusdriven processes for target detection. ACC and DLPFC are found to be active during executive control, whereas IPS is more active during bottom-up or surprise processing. For example, a number of neuroimaging studies using variants of the Stroop task have shown activation of the dorsal ACC and the DLPFC in tasks requiring subjects to respond to one dimension of a stimulus (e.g., ink color) rather than another more subjectively salient dimension (e.g., word meaning) conveying conflicting information (Liu, Banich, Jacobson, \& Tanabe, 2004, 2006; Fan, Flombaum, McCandliss, Thomas, \& Posner, 2003; MacDonald, Cohen, Stenger, \& Carter, 2000). Meta-analyses of neuroimaging studies have revealed that ACC plays an important role in executive control (Bush, Luu, \& Posner, 2000), that ACC and DLPFC functionally interact to support executive control (Ridderinkhof, Ullsperger, Crone,

\footnotetext{
${ }^{1}$ Mount Sinai School of Medicine, New York, NY, ${ }^{2}$ University of California at Berkeley, CA, ${ }^{3}$ Brain Trauma Foundation, New York, NY, ${ }^{4}$ Weill Medical College of Cornell University, New York, NY *These two authors made equal contribution to the current study.
}

various models in which different connections among ACC, DLPFC, and IPS were modulated by bottom-up stimulus-driven surprise and top-down conflict processing using Bayesian model selection procedures. The most optimal of these models incorporated contextual modulation that allowed processing of unexpected (surprising) targets to mediate the influence of the IPS over ACC and DLPFC and conflict processing to mediate the influence of ACC and DLPFC over the IPS. This result suggests that the IPS plays an initiative role in this network in the processing of surprise targets, whereas ACC and DLPFC interact with each other to resolve conflict through attentional modulation implemented via the IPS.

\& Nieuwenhuis, 2004), and that ACC, DLPFC, and posterior parietal cortex are involved in detection and/or resolution of conflict (Nee, Wager, \& Jonides, 2007). In addition, the IPS is activated in response to cues predicting target locations for bottom-up stimulus-driven responses (Corbetta \& Shulman, 2002). Although many theories have been proposed to account for the collaborative roles of these brain areas in the attentional control system (Botvinick, Cohen, \& Carter, 2004; Pessoa \& Ungerleider, 2004; Corbetta \& Shulman, 2002; Botvinick, Braver, Barch, Carter, \& Cohen, 2001), the exact role of each of these areas plays in such a complicated system and how they dynamically interact with each other remain to be unraveled (Dosenbach, Fair, Cohen, Schlaggar, \& Petersen, 2008; Rowe et al., 2007; Miller \& Cohen, 2001; Banich et al., 2000; Miller, 2000).

Several specific questions remain to be investigated. First, the distinct functions subserved by different brain regions of the fronto-parietal network are still not clear. One group of researchers proposed that ACC serves a central role in conflict detection, which in turn recruits participation of the DLPFC and IPS to resolve the conflict (Botvinick et al., 2001, 2004; Botvinick, Nystrom, Fissell, Carter, \& Cohen, 1999). Another group made the distinction that the "cingulo-opercular" network serves to maintain a stable set whereas the fronto-parietal network provides dynamic adjustment (Dosenbach et al., 2006, 2007, 2008). Although these theories provide useful frameworks to organize some empirical findings, increasing evidence from other studies suggests that there is a need to 
further investigate these two frameworks. Some even question the functional specificity of the brain regions of the attentional control network (Fellows \& Farah, 2005). For example, many studies have found that the lateral prefrontal and parietal cortices also play essential roles in establishing and maintaining task set and goal representations in a variety of tasks, including attentional control (Nee et al., 2007; Wager, Jonides, \& Reading, 2004; Banich et al., 2000; MacDonald et al., 2000), working memory (Rowe et al., 2007; Wager \& Smith, 2003; Rowe, Toni, Josephs, Frackowiak, \& Passingham, 2000), and task switching (Sylvester et al., 2003; Dove, Pollmann, Schubert, Wiggins, $\&$ von Cramon, 2000). Furthermore, evidence seems to suggest that medial prefrontal regions including ACC provide a more dynamic adjustment of attentional control on a trial-by-trial basis (Kerns et al., 2004; Botvinick et al., 1999), and activity in ACC attenuates to a greater degree than that of the DLPFC during the course of training of executive control tasks (Erickson et al., 2007; Milham, Banich, Claus, \& Cohen, 2003).

Second, both top-down task-driven and bottom-up stimulus-driven attentional control seem to commonly activate the fronto-parietal network. However, the unique functionality of each of these regions is still unclear. Previous studies have shown that this fronto-parietal network is recruited by top-down attentional control (Knight, 2007; Miller, 2000; Knight, Grabowecky, \& Scabini, 1995), which directs attentional resources toward certain aspects of stimulus processing and modulates activity in specific cortical areas responsible for such sensory processing (Hopfinger, Buonocore, \& Mangun, 2000; Kastner \& Ungerleider, 2000). For example, the DLPFC has been implicated in top-down attentional control during response anticipation (Fassbender, Foxe, \& Garavan, 2006; Liang, Bressler, Ding, Truccolo, \& Nakamura, 2002; Miller, 2000; Fuller \& Jahanshahi, 1999), the active maintenance of task goals and representations (Rowe et al., 2007; Miller \& Cohen, 2001; Banich et al., 2000) and top-down control of visual attention and response conflict (Yago, Duarte, Wong, Barcelo, \& Knight, 2004; Barcelo, Suwazono, \& Knight, 2000; Gehring \& Knight, 2000).

Similarly, studies have suggested that ACC, superior frontal cortex, and cortex along the IPS are involved in cued task set implementation and in holding information on-line during response anticipation (Dosenbach et al., 2006; Quintana, Wong, Ortiz-Portillo, Marder, \& Mazziotta, 2004; Petit, Courtney, Ungerleider, \& Haxby, 1998). In addition, prefrontal and parietal regions are involved in sustaining attention during response anticipation (Hopfinger et al., 2000; Desimone, 1996). A large-scale distributed system, including the fronto-parietal network, is involved in "directed" attention (Mesulam, 1981, 1999) and in directing both visual attention and eye movement to target locations (Corbetta, 1998). In a previous report, we also identified brain activity associated with response anticipation (following a cue to prepare vs. relax) and with response conflict (responding to a target surrounded by incongruent vs. congruent flankers) in a combined ERP and
fMRI study (Fan et al., 2007). We found that similar areas in the fronto-parietal network, including ACC, DLPFC, and IPS, were commonly activated by both response anticipation (cueing effect) and conflict processing (conflict effect).

On the other hand, stimulus-driven attentional processing elicited by low-frequency (surprising and/or unexpected) events also recruits the fronto-parietal network. For example, an fMRI study on perceiving patterns in a random sequence showed that violations of repeating patterns evoked activation in the $\mathrm{pFC}$, the posterior rostral ACC, the fronto-insular cortex, and the basal ganglia (Huettel, Mack, \& McCarthy, 2002). Both amplitude of the hemodynamic response in these regions and RT are associated with the length of the sequence before the violation. In anther fMRI study, participants were presented with cue cards and were asked to make a two-choice response to predict whether the next card would be higher or lower. Greater activation in ACC and fronto-insular cortex was associated with higher uncertainty (Critchley, Mathias, \& Dolan, 2001). In addition, the DLPFC and IPS have been shown to be related to information processing evoked by the occurrence of a high-information event (Strange, Duggins, Penny, Dolan, \& Friston, 2005).

Third, although some studies have started to differentiate the functions of these brain regions in the frontoparietal network, additional investigation of the functions of these regions in the context of effective connectivity is desired to clearly elucidate their dynamic interactions in controlling attention. The contributions of lateral prefrontal and parietal cortices and ACC to visual target responses have been shown to be dissociable such that the parietal cortex is related to the representation of responses and the lateral pFC and ACC are associated with the selection of responses from competing alternatives in a flanker task (Bunge, Hazeltine, Scanlon, Rosen, \& Gabrieli, 2002). A study using a similar paradigm and methods as those that we used in our study revealed that first the frontal cortex and then the parietal cortex are engaged during top-down attentional control (Grent-'tJong \& Woldorff, 2007). A resting state functional connectivity analysis revealed a fronto-parietal network that includes the DLPFC and the IPS, proposed to be involved in initiation and adaptation of control on a trial-by-trial basis, as well as another separate network that includes the dorsal ACC, the anterior insula/frontal operculum, and the anterior pFC, proposed to be involved in goal directed behavior through the stable maintenance of task sets (Dosenbach et al., 2007). In addition, a dynamic causal model (DCM) of effective connectivity during visual oddball processing (the surprise effect) showed significant connectivity from the ACC to the DLPFC and IPS (Brazdil, Mikl, Marecek, Krupa, \& Rektor, 2007).

To further understand distinct roles of and interactions among these brain regions under different task conditions, we examined the effective connectivity of ACC, DLPFC, and IPS within the fronto-parietal network during processing of surprise and conflict using DCM. We aimed 
to explore how the interaction between ACC and DLPFC is modulated by surprise and conflict effects. We hypothesized that surprise and conflict processing exert dissociable contextual modulations on the intrinsic connections among these regions, specifically that the surprise processing of targets would be mediated by the forward connections from the IPS to the DLPFC and ACC, whereas the conflict effect would be mediated by the backward connections from the DLPFC and ACC to the IPS.

\section{METHODS}

\section{Participants}

Eighteen healthy adults (mean age $=26$ years, range $=18-$ 59 years) participated in this experiment. Exclusion criteria for participation were the history of traumatic brain injury with loss of consciousness within the past year, acute intoxication, drug or alcohol abuse history, neurological or psychiatric diagnosis, and all contraindications for MRI (e.g., claustrophobia). A signed informed consent approved by the New York Presbyterian Hospital/Weill Medical College of Cornell University was obtained from each participant before the experiment. The results of conventional analysis of the data collected from these subjects were reported in a previous article (Fan et al., 2007).

\section{Experimental Design}

The response anticipation-response conflict task was designed to elicit processing related to response anticipation (in contrast to surprise target onset) and response conflict. In this task, a warning cue was followed by an imperative target after a cue target interval of $2.5 \mathrm{sec}$ in each trial. The target was an arrow flanked on both sides by either two arrows pointing in the same direction (congruent condition) or in the opposite direction (incongruent condition). There were four trial types: (1) no cue-no target (baseline), in which participants were shown no events but only the consistent appearance of a fixation cross; (2) no cuetarget (with congruent or incongruent flankers), in which an unexpected target was presented without cueing; (3) relax cue-no target, in which participants were shown a pair of triangles above and below fixation indicating that no target would be presented; and (4) ready cue-target (with congruent or incongruent flankers), in which participants were shown a pair of circles above and below fixation, informing participants to get ready to respond to the upcoming target.

Participants indicated the direction of the center arrow by pressing one key with the left hand for the left direction and another key with the right hand for the right direction. Different trial types were pseudorandomized across eight runs of 32 trials so that each trial type had an equal probability of appearing before and after other specific trial. Each run lasted $288 \mathrm{sec}$. The first and the last 16 sec of each run were rest periods. RT and accuracy were recorded for each trial.

\section{Data Acquisition}

Participants were scanned using a General Electric Signa 3.0 Tesla MRI scanner (General Electric Medical Systems, Milwaukee, WI). The blood oxygen level-dependent (BOLD) responses were measured by a spiral in-and-out sequence with parameters as follows: $\mathrm{TR}=2500 \mathrm{msec}$, $\mathrm{TE}=40 \mathrm{msec}$, field of view $=220 \mathrm{~mm}$, flip angle $=90^{\circ}$, matrix $=64 \times 64$, thirty-two 5-mm-thick (no gap) axial slices with an in-plane resolution of $3.4375 \times 3.4375 \mathrm{~mm}^{2}$, parallel to the anterior commissure-posterior commissure line. The total number of volumes for each run was 116 . After one run of the practice trials, six runs of functional images were acquired. Other details of experimental design and data acquisition can be found in our previous report (Fan et al., 2007).

\section{Statistical Parametric Mapping}

The data analyses previously reported were performed using SPM2 (Wellcome Department of Imaging Neuroscience, London, UK). To facilitate the application of DCM (Friston, Harrison, \& Penny, 2003) analysis and Bayesian model selection (BMS) (Penny, Stephan, Mechelli, \& Friston, 2004), we reanalyzed the data in a slightly different way using SPM5. Within each individual subject, all six functional runs were concatenated to provide an adequate number of data points so that the model parameters could be better estimated and so that our ability to discriminate models with different complexity using BMS could be enhanced (Penny et al., 2004). The first two volumes of each run were discarded. The remaining functional scans were realigned to the first volume, coregistered to the T1 image, normalized to a standard template (Montreal Neurological Institute; MNI), resampled to $2 \times 2 \times 2 \mathrm{~mm}^{3}$ voxels, and spatially smoothed with an $8 \times 8 \times 8 \mathrm{~mm}^{3}$ FWHM Gaussian kernel.

A general linear model was constructed for each participant to characterize the hemodynamic responses under different experimental conditions. Regressors were created by convolving a train of delta functions representing the cue and the target events with the default SPM basis function, which consists of a synthetic hemodynamic response composed of two gamma functions (Friston et al., 1998). The general linear model included four regressors: (1) "all cues," which was time locked to cue events and modeled to estimate the cue-related response but was not of interest in this study; (2) "all targets," which was target locked to the presentation of all target types; (3) "no-cue targets," which was target locked to the presentation of targets that were not preceded by cues; and (4) "incongruent targets," which was target locked to all incongruent targets. The cue-related visual response was assumed to be regressed out by Regressor 1. Regressor 2 was intended to 
serve as a driving input and a baseline to which all modulatory effects would be referenced. Regressor 3 represented the main effect of surprise and was modeled because we found in a previous study that uncued targets activated the fronto-parietal networks more than cued targets. Regressor 4 represented the main effect of conflict in the DCM analysis. Because we did not find significant cue by target interaction in our previous study, we did not include an interaction regressor in the current study. In addition to Regressor 1, other regressors of no interest included estimated motion parameters and regressors accounting for signal differences across runs. A 128-sec temporal high-pass filter was applied to the data to remove low-frequency noise, and a first-order autoregressive model was used to remove serial correlations.

The specific effects of conditions of all cues, all targets, no-cue targets, and incongruent targets for each subject were estimated. The resultant contrast images of each participant were entered into a second-level randomeffects group analysis. The combination of an uncorrected $p$ value of .01 for the intensity threshold of each voxel and a minimum cluster extent threshold of 85 contiguous resampled voxels $\left(2 \times 2 \times 2 \mathrm{~mm}^{3}\right)$ was used to correct for multiple voxel comparisons and yielded a corrected threshold of $p<.05$ for each condition, as determined by a Monte Carlo simulation (Slotnick \& Schacter, 2004). For the volume of interest (VOI) in the DLPFC, we adopted a less strict threshold of an uncorrected $p$ value of .05 with an extent threshold of 200 voxels because activation in this structure could not be found using the stricter threshold.

\section{Region Selection and Time Series Extraction}

The selection of subject-specific VOIs in ACC, DLPFC, and IPS was based on the group maxima of random-effects analysis for the all targets regressor. Region-specific time series were generated by calculating the principle eigenvariate from all voxels passing significance within $4 \mathrm{~mm}$ of the local maximum identified in statistical parametric maps in each individual subject. To ensure the comparability of the location of the VOI across subjects, we combined anatomic and functional constraints as suggested by previous studies (Heim et al., 2007; Stephan, Harrison, et al., 2007); that is, each subject-specific local maximum was required to be located within the same gyrus as the group maximum and survive an uncorrected threshold of $p<.05$. With these criteria, we could not extract time series in 4 of the 18 subjects due to lack of activation in one of these three areas. These four subjects were excluded from the DCM analysis. In addition, for simplicity of the model setup, we focused on regions in the right hemisphere.

\section{Dynamic Causal Modeling}

DCM treats the brain as a dynamic input-state-output system that is subject to experimentally controlled manipu- lations (Friston et al., 2003; Mechelli, Price, Noppeney, \& Friston, 2003). Such manipulations influence the system in terms of input that can either elicit responses through direct influence on specific regions ("driving input") or that can change the strength of coupling among regions ("modulatory input"). The state variables represent the neuronal activity in each region, which cannot be observed directly using fMRI. The outputs are the regional hemodynamic responses, which are connected to the neuronal state variables using a biophysically validated forward model of hemodynamic responses (Stephan, Weiskopf, Drysdale, Robinson, \& Friston, 2007). The central idea of DCM is to model changes in the states such that the predicted BOLD signal corresponds as closely as possible to the observed BOLD time series (Stephan, Penny, Marshall, Fink, \& Friston, 2005). There are three sets of parameters in DCM: (1) driving input, which mediates influence of external input on the neuronal states; (2) intrinsic connection, which represents coupling between neuronal states in different regions; and (3) modulatory input, which reflects the context-dependent changes in the coupling between regions.

\section{Construction of the Models}

Due to the fact that effective connectivity as inferred by DCM does not necessarily mandate monosynaptic anatomical connectivity and because of the lack of detailed anatomical knowledge of connectivity structure, subjectspecific models were first constructed with all three regions fully and reciprocally connected. The effect of all targets was modeled as the driving input entering into the model through the IPS and propagating from the IPS to other regions through intrinsic connections. Given that it is widely believed that ACC and DLPFC interact to support attentional control, we assumed that the surprise and the conflict effects modulate the bidirectional connection between ACC and DLPFC in all models. The primary focus of this study was to look at how the surprise and the conflict effects influence the connections between the IPS and the ACC and between the IPS and the DLPFC. Here, Model 1 embodied our main hypothesis, and Models 2, 3, and 4 served as alternative models (Figure 2). In Model 1, the surprise effect modulated the forward connections from the IPS to the ACC and DLPFC, and the conflict effect modulated the backward connections from the ACC and DLPFC to the IPS. In Model 2, we had the surprise effect modulate the backward connections from the ACC and DLPFC to the IPS and the conflict effect to modulate the forward connections from the IPS to the ACC and DLPFC. In Model 3, we allowed the surprise and the conflict effects to modulate the backward connections from the ACC and DLPFC to the IPS. Finally, in Model 4, modulatory inputs of the surprise and the conflict effects were allowed to modulate all connections. 


\section{Model Selection and Parameter Testing}

In this study, BMS was used to find the optimal model among the four specified. The implementation of BMS in SPM5 uses two approximations, the Bayesian information criterion (BIC) and the Aikake information criterion (AIC), to estimate model evidence (Penny et al., 2004). Ratios of model evidences from two models computed using either BIC or AIC are termed Bayes factors (BF) and provide information regarding which of the two models are favored. For example, when comparing hypothetical models A and B, a BF greater than 3 (3-20) is interpreted as a positive evidence for model A. Positive evidence indicated by a BF calculated using BIC biases toward selection of simple models, whereas positive evidence indicated by a BF calculated using AIC biases toward selection of better fit models. To choose a model that is both parsimonious and accurate, we selected models in which the lower of the two BFs exceeded 3; that is, both provided positive evidence, as adopted in a previous study (Penny et al., 2004). When comparing a pair of models across a group of subjects, a group Bayes factor (GBF) was computed as the product of each subject's individual BF (Stephan \& Penny, 2006). Because GBF is sensitive to the effect of outliers, we computed the ratio of subjects with positive evidence for one model versus those having positive evidence for the other, the positive evidence ratio, to determine if the results we obtained were due to a subject with an extreme BF (Stephan \& Penny, 2006). Once the optimal model was identified, the group average of the optimal model was obtained by running DCM average function of SPM5, which corresponds to a Bayesian fixed-effects analysis. We report those parameter estimates for which there was at least a probability of $90 \%$ that their posterior mean deviated from zero.

\section{RESULTS}

RTs were significantly longer for the uncued than the cued targets and for the incongruent than the congruent targets. The accuracy was lower for the uncued than the cued targets and for the incongruent than the congruent targets. The interactions between the cue and the conflict for both RT and accuracy were not significant. Additional details on the behavioral results can be found in our previous report (Fan et al., 2007).

\section{Conventional fMRI Results}

The effect of all targets activated the occipital areas [Brodmann's areas (BAs) 18 and 19], the temporal areas (BA 37), the frontal pole (BA 10) extending into the DLPFC (BA 46), the ACC (BA 24), the insula, and the IPS (BA 7) extending into superior parietal lobule, among other regions (see Table 1 and Figure 1). Most of the activation was distributed bilaterally. Notably, activation of ACC,
DLPFC, and IPS elicited by the surprise effect (Table 2) and the conflict effect (Table 3) estimated with the data sets concatenated across multiple runs showed similar patterns of activation as those found in the original report (Fan et al., 2007).

\section{DCM Results}

Table 4 shows the coordinates of the VOIs in each individual subject and their means as well as the peak coordinates from the group random-effects analysis.

Table 1. Activation Associated with the All Target Regressor

\begin{tabular}{|c|c|c|c|c|c|c|}
\hline Region & $B A$ & $x$ & $y$ & $z$ & Voxel & $Z$ \\
\hline R Superior temporal pole & 38 & 54 & 10 & -4 & 1503 & 5.32 \\
\hline R Insula ${ }^{\mathrm{a}}$ & & 36 & 16 & 2 & & 4.85 \\
\hline L Postcentral gyrus $^{\mathrm{b}}$ & 3 & -48 & -26 & 60 & 3641 & 5.09 \\
\hline L Inferior parietal lobule & 40 & -48 & -24 & 42 & & 4.72 \\
\hline R Cerebellum $^{\mathrm{c}}$ & & 26 & -60 & -16 & 1915 & 4.70 \\
\hline R Inferior temporal gyrus & 37 & 42 & -62 & -8 & & 4.38 \\
\hline R Supramarginal gyrus & 40 & 60 & -18 & 22 & 814 & 4.50 \\
\hline R ACC & 24 & 2 & 24 & 32 & 1201 & 4.40 \\
\hline L SMA & 32 & -4 & 12 & 46 & & 4.29 \\
\hline L Rolandic operculum $^{\mathrm{d}}$ & 6 & -56 & 6 & 6 & 2018 & 4.39 \\
\hline L Pallidum & & -14 & 10 & -2 & & 4.01 \\
\hline L Inferior occipital gyrus & 37 & -48 & -66 & -2 & 448 & 4.12 \\
\hline L Middle temporal gyrus & 37 & -48 & -58 & 4 & & 3.50 \\
\hline L Middle occipital gyrus & 19 & -42 & -80 & 10 & & 2.99 \\
\hline R Postcentral gyrus & 3 & 44 & -34 & 66 & 887 & 3.88 \\
\hline R Intraparietal sulcus ${ }^{\mathrm{e}}$ & 7 & 24 & -62 & 60 & & 3.09 \\
\hline R Cuneus & 18 & 10 & -68 & 24 & 94 & 3.73 \\
\hline R Pallidum & & 16 & 8 & 0 & 285 & 3.52 \\
\hline R Putamen & & 26 & 2 & 2 & & 2.46 \\
\hline R Middle frontal gyrus ${ }^{\mathrm{f}}$ & $10 / 46$ & 36 & 56 & 10 & 318 & 3.43 \\
\hline
\end{tabular}

$\mathrm{R}=$ right $\mathrm{L}=$ left $\mathrm{BA}=$ Brodmann area; $\mathrm{ACC}=$ anterior cingulate cortex; SMA = supplementary motor area.

${ }^{\mathrm{a}}$ Extends into the $\mathrm{R}$ rolandic operculum (BA 6) and the R inferior frontal gyrus (BA 44).

${ }^{b}$ Extends into the L supramarginal gyrus (BA 40), the L precentral gyrus (BA 6), the $\mathrm{L}$ superior frontal gyrus (BA 6), and the $\mathrm{L}$ middle frontal gyrus (BA 8).

${ }^{\circ}$ Extends into the $\mathrm{R}$ fusiform gyrus (BA 37).

${ }^{\mathrm{d}}$ Extends into the $\mathrm{L}$ insular cortex, the $\mathrm{L}$ thalamus, and the $\mathrm{L}$ putamen. ${ }^{e}$ Extends into the superior parietal lobules.

${ }^{\mathrm{f}}$ This cluster, within a VOI in the DLPFC, survived the intensity threshold of an uncorrected $p$ value of .05 and an extent threshold of 200 voxels. A local maximum within this cluster was used as the VOI of the DLPFC to extract the time series. 


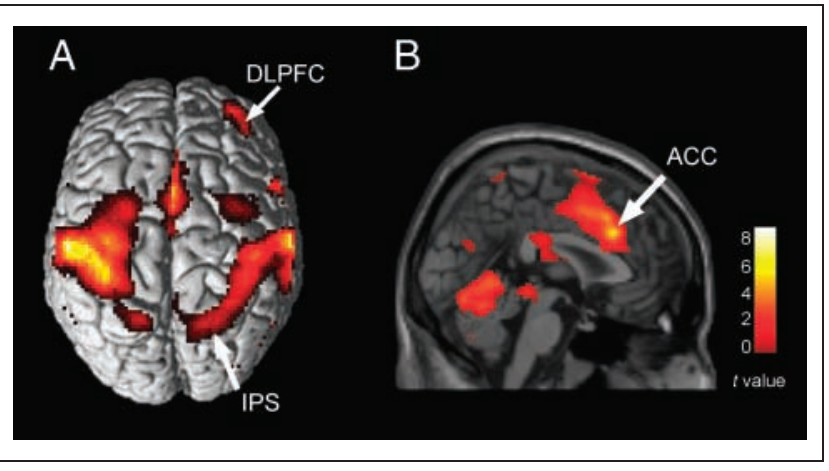

Figure 1. Brain activation elicited by the all targets shown on a surface rendering (A) and cross-sectional view (B). ACC, DLPFC, and IPS were selected as VOIs for the DCM analysis. To show the activated cluster in the DLPFC, this figure was generated using an uncorrected threshold of $p<.05$ with an extent threshold of 200 voxels. See Table 1 for other activated regions.

The average coordinates $(\mathrm{mm})$ in the MNI template space were 2, 17, 36 for the ACC; 38, 49, 23 for the DLPFC; and $27,-58,62$ for the IPS. The mean \pm SD distances of individual VOIs from the group peak coordinates were $11 \pm$ $5 \mathrm{~mm}$ for the ACC, $11 \pm 6 \mathrm{~mm}$ for the DLPFC, and $9 \pm$ $5 \mathrm{~mm}$ for the IPS. Table 5 summarizes the individual BFs and the GBF for pairwise model comparison between Model 1 and all other alternative models. When comparing Model 1 with other models across participants, the GBF was greater than $10^{14}$, at least 8 of 14 subjects showed positive evidence in favor of Model 1 , and no single $\mathrm{BF}$ provided positive evidence for any other models. These findings provided strong evidence in favor of Model 1 over the other models for the current data. In addition, as Models 1, 2, and 3 had the same number of free parameters
Table 2. Activation Associated with the Surprise Effect (by the Uncued Target)

\begin{tabular}{|c|c|c|c|c|c|c|}
\hline Region & $B A$ & $x$ & $y$ & $z$ & Voxel & $Z$ \\
\hline R ACC & 32 & 8 & 18 & 42 & 955 & 5.12 \\
\hline L ACC & 32 & -8 & 18 & 40 & & 3.20 \\
\hline L Precentral gyrus & 44 & -46 & 6 & 32 & 695 & 4.54 \\
\hline L Inferior frontal gyrus & 44 & -46 & 14 & 20 & & 2.62 \\
\hline $\mathrm{R}$ Precentral gyrus ${ }^{\mathrm{a}}$ & 6 & 42 & -14 & 56 & 1804 & 4.01 \\
\hline R Postcentral gyrus & 3 & 46 & -14 & 40 & & 3.97 \\
\hline L Middle occipital gyrus & 19 & -28 & -86 & 30 & 133 & 3.95 \\
\hline R Middle cingulate cortex & 31 & 10 & -16 & 44 & 222 & 3.90 \\
\hline $\mathrm{R}_{\text {Calcarine fissure }}{ }^{\mathrm{b}}$ & 17 & 10 & -62 & 18 & 1878 & 3.83 \\
\hline R Cuneus & 7 & 8 & -78 & 40 & & 3.67 \\
\hline L Calcarine fissure & 17 & -18 & -66 & 14 & & 3.51 \\
\hline L Inferior frontal gyrus ${ }^{c}$ & 45 & -42 & 46 & 6 & 1199 & 3.56 \\
\hline L Middle frontal gyrus & 46 & -34 & 48 & 24 & & 3.55 \\
\hline L Cuneus & 19 & -16 & -74 & 38 & 115 & 3.00 \\
\hline L Superior occipital gyrus & 19 & -12 & -80 & 46 & & 2.71 \\
\hline R Lingual gyrus & 19 & 16 & -48 & 0 & 135 & 2.91 \\
\hline L Insula & & -30 & 22 & -10 & 112 & 2.78 \\
\hline
\end{tabular}

$\mathrm{R}=$ right $; \mathrm{L}=$ left $\mathrm{BA}=$ Brodmann area; $\mathrm{ACC}=$ anterior cingulate cortex.

${ }^{\mathrm{a}}$ Extends into the R inferior frontal gyrus (BA 45).

${ }^{\mathrm{b}}$ Extends into the L lingual gyrus (BAs 27, 37, and 18), the L cuneus (BA 7), the $\mathrm{L}$ cerebellum, and the $\mathrm{L}$ fusiform gyrus (BA 37).

${ }^{c}$ Extends into the $\mathrm{L}$ middle frontal gyrus (BAs 45 and 10), the $\mathrm{L}$ inferior frontal gyrus (BA 47)

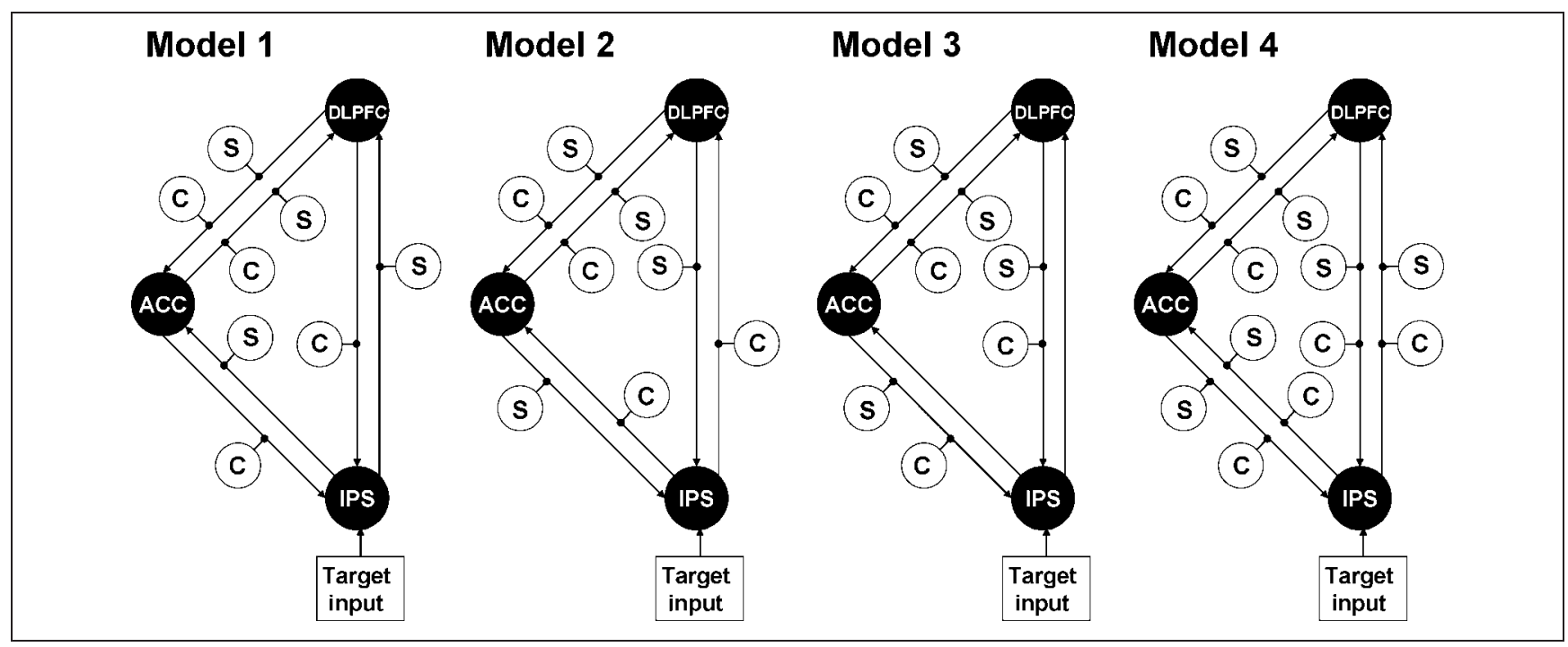

Figure 2. Diagrammatic representation of the four models evaluated and compared in this study. Black-filled circles indicate the regions included in DCM. Arrows represent the directional intrinsic connections between the regions. White circles indicate the modulatory inputs of the surprise and the conflict effects. The rectangles represent the driving input of all targets entering into the network via the IPS. S = surprise modulatory effect; $\mathrm{C}=$ conflict modulatory effect. 
Table 3. Activation Associated with the Conflict Effect (by the Target with Incongruent Flankers)

\begin{tabular}{lrrrrrr}
\hline Region & $B A$ & $x$ & $y$ & $z$ & Voxel & $Z$ \\
\hline L ACC & 32 & -8 & 24 & 38 & 1662 & 4.04 \\
R Middle cingulate cortex & 31 & 12 & -18 & 48 & & 3.55 \\
R ACC & 24 & 4 & 0 & 34 & & 3.29 \\
R Postcentral gyrus & 4 & 32 & -22 & 50 & 1803 & 3.50 \\
R Superior frontal gyrus & 9 & 20 & 32 & 42 & & 3.50 \\
R Middle frontal gyrus & 8 & 28 & 16 & 58 & & 3.42 \\
L Thalamus & & -8 & -24 & 14 & 111 & 3.45 \\
L Inferior frontal gyrus & 44 & -58 & 12 & 26 & 253 & 3.38 \\
L Precentral gyrus & 6 & -54 & 8 & 34 & & 3.21 \\
L Inferior parietal lobule & 40 & -34 & -48 & 50 & 89 & 3.03
\end{tabular}

$\mathrm{R}=$ right $; \mathrm{L}=$ left $\mathrm{BA}=$ Brodmann area; $\mathrm{ACC}=$ anterior cingulate cortex.

${ }^{a}$ Extends into the precentral gyrus (BAs 4 and 6), the middle frontal gyrus (BAs 46 and 8), and the inferior frontal gyrus (BA 46).

Table 4. MNI Coordinates (mm) for the VOIs Included in DCM for Each Subject

\begin{tabular}{|c|c|c|c|c|c|c|c|c|c|}
\hline \multirow[b]{2}{*}{ Subject } & \multicolumn{3}{|c|}{$A C C$} & \multicolumn{3}{|c|}{$D L P F C$} & \multicolumn{3}{|c|}{ IPS } \\
\hline & $x$ & $y$ & $z$ & $x$ & $y$ & $z$ & $x$ & $y$ & $z$ \\
\hline 01 & 2 & 28 & 38 & 42 & 40 & 28 & 20 & -64 & 62 \\
\hline 02 & 2 & 18 & 32 & 44 & 50 & 18 & 26 & -62 & 56 \\
\hline 03 & 0 & 6 & 42 & 42 & 50 & 24 & 32 & -50 & 62 \\
\hline 04 & 4 & 12 & 44 & 24 & 62 & 66 & 24 & -62 & 66 \\
\hline 05 & 4 & 6 & 40 & 44 & 36 & 30 & 24 & -62 & 60 \\
\hline 06 & -2 & 20 & 40 & 36 & 56 & 18 & 26 & -54 & 64 \\
\hline 07 & -2 & 16 & 48 & 38 & 42 & 26 & 20 & -64 & 64 \\
\hline 08 & 4 & 14 & 34 & 38 & 52 & 26 & 32 & -52 & 64 \\
\hline 09 & 4 & 14 & 36 & 30 & 58 & 24 & 32 & -62 & 52 \\
\hline 10 & 4 & 28 & 30 & 34 & 46 & 38 & 34 & -48 & 66 \\
\hline 11 & 6 & 24 & 32 & 40 & 44 & 26 & 22 & -60 & 66 \\
\hline 12 & 6 & 24 & 26 & 38 & 58 & 14 & 30 & -62 & 68 \\
\hline 13 & 4 & 14 & 28 & 28 & 56 & 20 & 30 & -56 & 62 \\
\hline 14 & -2 & 16 & 42 & 30 & 50 & 22 & 24 & -54 & 58 \\
\hline Mean & 2 & 17 & 36 & 38 & 49 & 23 & 27 & -58 & 62 \\
\hline RFX & 2 & 24 & 32 & 40 & 44 & 26 & 24 & -62 & 60 \\
\hline
\end{tabular}

The coordinates refer to the subject-specific local maximum of the all targets effect, at which fMRI time series are extracted for the DCM analysis. The cross-subject means are rounded. The bottom row lists the coordinates of the group maximum in group random-effects (RFX) analysis.
Table 5. BFs, GBF, and Positive Evidence Ratios (PER) for the Comparison of Model 1 with Models 2, 3, and 4

\begin{tabular}{llcc}
\hline \multirow{2}{*}{ Subjects } & \multicolumn{3}{c}{ Model 1 vs. } \\
\cline { 2 - 4 } 01 & Model 2 & Model 3 & Model 4 \\
\hline 02 & $5.33 \mathrm{E}+08$ & $5.31 \mathrm{E}+08$ & 162.00 \\
03 & 13.59 & 13.36 & 49.54 \\
04 & 1.66 & 7.21 & 8.39 \\
05 & 6.24 & 10.90 & 21.14 \\
06 & 7.03 & 4.79 & 51.99 \\
07 & 1.00 & 1.00 & 54.59 \\
08 & 0.90 & 0.38 & 45.03 \\
09 & 4.78 & 18.68 & 37.72 \\
10 & 0.96 & 1.02 & 636.60 \\
11 & 1.04 & 1.06 & 54.67 \\
12 & 5.46 & 2.41 & 30.40 \\
13 & 1.00 & 1.00 & 54.50 \\
14 & 3.00 & 4.13 & 35.12 \\
GBF & 3.44 & 3.56 & 51.95 \\
PER & $1.28 \mathrm{E}+14$ & $7.34 \mathrm{E}+14$ & $8.45 \mathrm{E}+22$ \\
\hline
\end{tabular}

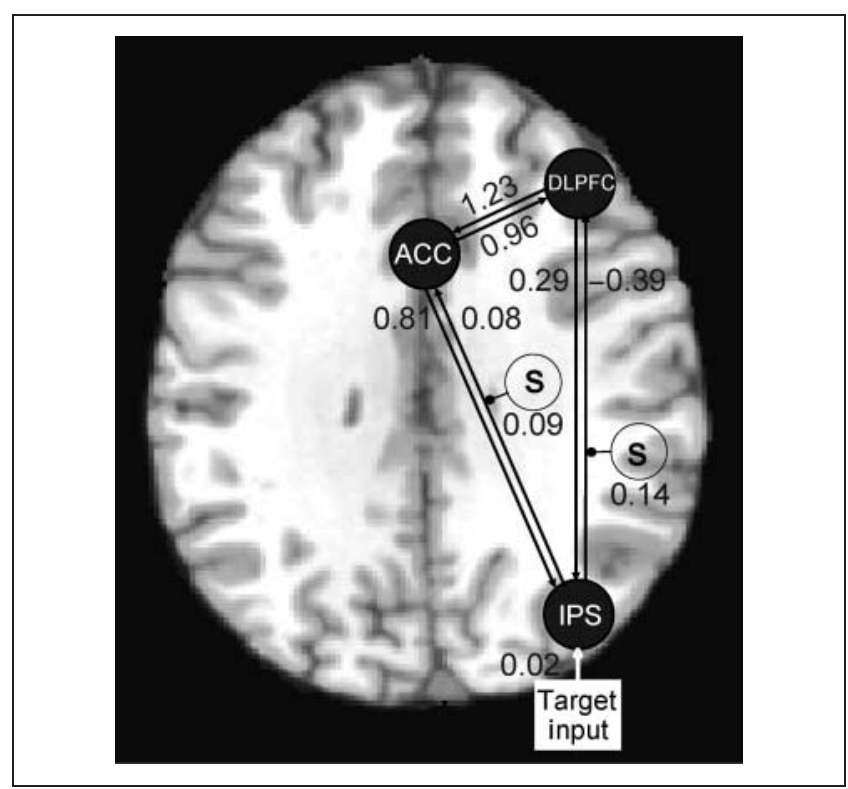

Figure 3. Significant parameters of Model 1 (the most optimal among those tested) in the group analysis superimposed on an axial brain slice. The values are parameter estimates of the group average of Model 1 across subjects based on the Bayesian analysis. For clarity, this figure does not show the nonsignificant parameters of Model 1 in the group analysis. For other details, see Figure 2 and Table 5. $\mathrm{S}=$ surprise modulatory effect. 
(including intrinsic connections, modulatory effects, and driving input), the difference in model evidence was solely due to model fit and not model complexity.

Finally, we examined whether the model parameters were significant in the most optimal model tested (Model 1). Figure 3 shows parameter estimates for which there was at least a probability of $90 \%$ that their posterior means deviated from zero, based from the group average of the optimal model. The posterior means of all bidirectional intrinsic connections among ACC, DLPFC, and IPS as well as the driving input to the IPS significantly deviated from zero. The posterior means of modulatory effects obtained by the surprise effect on the forward connections from the IPS to the ACC and DLPFC were significantly greater than zero. Notably, compared with the intrinsic connections from the IPS to the ACC and DLPFC, the modulatory influences induced by the surprise effect led to a change in connection strength by $113 \%$ and $36 \%$, respectively.

\section{DISCUSSION}

The current findings elucidated the distinct and interactive roles of ACC, DLPFC, and IPS in bottom-up stimulusdriven and top-down control systems by examining the modulatory effects of surprise and conflict processing on the fronto-parietal network. Previous studies on topdown control have indicated that the fronto-parietal network is commonly activated by a variety of manipulations of attentional control, including task set and goal representation (Rowe et al., 2007; Dosenbach et al., 2006; Miller \& Cohen, 2001; Banich et al., 2000), interference resolution (Nee et al., 2007; Liu et al., 2004; Fan et al., 2003; Botvinick et al., 1999, 2001), attention shifting (Kincade, Abrams, Astafiev, Shulman, \& Corbetta, 2005; Wager et al., 2004; Luks, Simpson, Feiwell, \& Miller, 2002; Corbetta et al., 1998), and response inhibition (Garavan, Hester, Murphy, Fassbender, \& Kelly, 2006; Liu et al., 2006; Wager et al., 2005) as well as working memory (Wager \& Smith, 2003; Corbetta, Kincade, \& Shulman, 2002; Cabeza \& Nyberg, 2000; Rowe et al., 2000). However, the specific roles of the brain regions involved in this top-down control system and how the interaction among these brain regions is modulated by various factors of attentional control have not been resolved.

In attempt to reconcile mixed results and go beyond the functional specificity of the brain regions involved in attentional control, many studies have begun to investigate the functional and effective connectivity among these regions using various connectivity analysis approaches (Dosenbach et al., 2008; Fan, Hof, Guise, Fossella, \& Posner, 2008; Brazdil et al., 2007; He et al., 2007; Margulies et al., 2007; Erickson, Ringo Ho, Colcombe, \& Kramer, 2005). Some have relied on spontaneous brain activity during the resting state, and few have studied the dynamics of the attentional control network under the context of attentional modulation. In a recent study, Brazdil et al. (2007) compared several connectivity models among brain regions of the fronto-parietal network using the DCM analysis of a visual oddball task. In that task, subjects were asked to count an infrequent target ("XXXXX" string, $6.25 \%$ ) out of a series of standard stimuli ("OOOOO" string, 93.75\%). They identified significant ACC, DLPFC, and IPS activation in response to the target stimuli and constructed different connectivity models among these regions using DCM. Because they did not have any modulatory effect, they focused more on the directional connectivity among these regions and tested the models with driving input either at the ACC or IPS. Through comparing the BFs across different models, they suggested that there exists bidirectional information flow among these regions, which signifies a parallel but distinct fronto-parietal network.

The current study went beyond modeling the intrinsic connections among brain regions in the fronto-parietal network and aimed to reveal the dynamics of these connections under the modulation of surprise and conflict processes. To test the hypothesis that these processes exert distinct modulation on the same attentional control network, we constructed a model (Model 4) in which three regions of the fronto-parietal network are fully and bidirectionally connected with each of the intrinsic connections modulated by both the surprise and the conflict effects. We then made more restricted and simpler models by removing some of the contextual modulation. In particular, we were interested in testing different models in which the surprise and the conflict effects differentially modulate the forward connections from the IPS to the ACC and DLPFC or the backward connections from the ACC and DLPFC to the IPS (Models 1 and 2). More specifically, we assumed that in Model 1, the surprise effect only affects the forward information flow from the IPS to the ACC and DLPFC, whereas the conflict effect only modulates the backward information flow from the ACC and DLPFC to the IPS. In Model 2, the surprise and the conflict modulatory effects on the forward and the backward connections were reversed from those in Model 1. Model 3 also serves as a comparison for the above models because it has the same complexity as Models 1 and 2 and only assumes modulatory effects on the backward connections from the frontal regions to the parietal cortex.

We found positive evidence to support that Model 1 is the most optimal among those tested. These findings have two implications. First, given the seemingly overlapping activation patterns elicited by both the surprise and the conflict effects in the fronto-parietal network, these two effects exert distinct modulation on the intrinsic connections among different brain regions. Both Models 3 and 4 assume the same modulation of surprise and conflict effects, yet the evidence is in favor of Model 1. This result cannot be attributed to favorable model evidence in support of Model 1 due to its simplicity because Models 1 and 3 have the same level of complexity in terms of model structure and free parameters. Second, evidence in favor of 
Model 1 over Model 2-given their same complexityindicates that the surprise effect exerts distinct modulation on the forward connection from the IPS to frontal areas, whereas the conflict effect modulates the backward connection from frontal areas to the IPS. This finding is consistent with a recent ERP study, which indicated the role of the IPS in initiating top-down attentional control (Green \& McDonald, 2008). Furthermore, these findings underscore the importance of using DCM to examine networks underlying cognitive functions; our analysis revealed that contextual changes altered the effective connectivity of the IPS independent of its level of activity. This important aspect of the fronto-parietal network cannot be revealed by examination of the functional activation results alone.

Group analysis on the model parameters of the optimal model (Model 1) revealed that there exist significant intrinsic bidirectional connections across all three regions. In accordance with previous studies (Heim et al., 2007; Stephan, Marshall, Penny, Friston, \& Fink, 2007), we also found that at least some (albeit not all) of the connections in the optimal model showed fairly robust modulatory effects compared with the fixed connection strengths. The forward connections between the IPS and the two frontal regions were significantly modulated by the surprise effect. However, the group average failed to reveal significant conflict-related modulation on those intrinsic connections, despite the strong evidence in favor of Model 1 over the other models. Nonetheless, these findings are in favor of the reciprocal relationship between the DLPFC and ACC and their shared influence (e.g., the surprise effect) by the posterior areas of the brain (e.g., IPS) in the attentional control system.

\section{Acknowledgments}

The Cognitive and Neurobiological Research Consortium in Traumatic Brain Injury research was supported by the James S. McDonnell Foundation through a collaborative grant to the Brain Trauma Foundation. Support for this work was also provided by NARSAD and NIH (MH083164) to J. F. and by NIH (NINDS NS21135) to R. T. K.

Reprint requests should be sent to Jin Fan, Laboratory of Neuroimaging, Department of Psychiatry, Mount Sinai School of Medicine, One Gustave L. Levy Place, Box 1230, New York, NY 10029, or via e-mail: Jin.Fan@mssm.edu.

\section{REFERENCES}

Banich, M. T., Milham, M. P., Atchley, R. A., Cohen, N. J., Webb, A., Wszalek, T., et al. (2000). Prefrontal regions play a predominant role in imposing an attentional "set": Evidence from fMRI. Brain Research. Cognitive Brain Research, 10, $1-9$.

Barcelo, F., Suwazono, S., \& Knight, R. T. (2000). Prefrontal modulation of visual processing in humans. Nature Neuroscience, 3, 399-403.

Botvinick, M. M., Braver, T. S., Barch, D. M., Carter, C. S., \& Cohen, J. D. (2001). Conflict monitoring and cognitive control. Psychological Review, 108, 624-652.

Botvinick, M. M., Cohen, J. D., \& Carter, C. S. (2004).
Conflict monitoring and anterior cingulate cortex: An update. Trends in Cognitive Sciences, 8, 539-546.

Botvinick, M. M., Nystrom, L. E., Fissell, K., Carter, C. S., \& Cohen, J. D. (1999). Conflict monitoring versus selection-for-action in anterior cingulate cortex. Nature, 402, 179-181.

Brazdil, M., Mikl, M., Marecek, R., Krupa, P., \& Rektor, I. (2007). Effective connectivity in target stimulus processing: A dynamic causal modeling study of visual oddball task. Neuroimage, 35, 827-835.

Bunge, S. A., Hazeltine, E., Scanlon, M. D., Rosen, A. C., \& Gabrieli, J. D. (2002). Dissociable contributions of prefrontal and parietal cortices to response selection. Neuroimage, 17, 1562-1571.

Bush, G., Luu, P., \& Posner, M. I. (2000). Cognitive and emotional influences in anterior cingulate cortex. Trends in Cognitive Sciences, 4, 215-222.

Cabeza, R., \& Nyberg, L. (2000). Imaging cognition II: An empirical review of 275 PET and fMRI studies. Journal of Cognitive Neuroscience, 12, 1-47.

Corbetta, M. (1998). Frontoparietal cortical networks for directing attention and the eye to visual locations: Identical, independent, or overlapping neural systems? Proceedings of the National Academy of Sciences, U.S.A., 95, 831-838.

Corbetta, M., Akbudak, E., Conturo, T. E., Snyder, A. Z., Ollinger, J. M., Drury, H. A., et al. (1998). A common network of functional areas for attention and eye movements. Neuron, 21, 761-773.

Corbetta, M., Kincade, J. M., \& Shulman, G. L. (2002). Neural systems for visual orienting and their relationships to spatial working memory. Journal of Cognitive Neuroscience, 14, 508-523.

Corbetta, M., \& Shulman, G. L. (2002). Control of goal-directed and stimulus-driven attention in the brain. Nature Reviews Neuroscience, 3, 201-215.

Critchley, H. D., Mathias, C. J., \& Dolan, R. J. (2001). Neural activity in the human brain relating to uncertainty and arousal during anticipation. Neuron, 29, 537-545.

Desimone, R. (1996). Neural mechanisms for visual memory and their role in attention. Proceedings of the National Academy of Sciences, U.S.A., 93, 13494-13499.

Dosenbach, N. U., Fair, D. A., Cohen, A. L., Schlaggar, B. L., \& Petersen, S. E. (2008). A dual-networks architecture of top-down control. Trends in Cognitive Sciences, 12, 99-105.

Dosenbach, N. U., Fair, D. A., Miezin, F. M., Cohen, A. L., Wenger, K. K., Dosenbach, R. A., et al. (2007). Distinct brain networks for adaptive and stable task control in humans. Proceedings of the National Academy, U.S.A., 104, 11073-11078.

Dosenbach, N. U., Visscher, K. M., Palmer, E. D., Miezin, F. M., Wenger, K. K., Kang, H. C., et al. (2006). A core system for the implementation of task sets. Neuron, 50, 799-812.

Dove, A., Pollmann, S., Schubert, T., Wiggins, C. J., \& von Cramon, D. Y. (2000). Prefrontal cortex activation in task switching: An event-related fMRI study. Brain Research. Cognitive Brain Research, 9, 103-109.

Erickson, K. I., Colcombe, S. J., Wadhwa, R., Bherer, L., Peterson, M. S., Scalf, P. E., et al. (2007). Training-induced functional activation changes in dual-task processing: An fMRI study. Cerebral Cortex, 17, 192-204.

Erickson, K. I., Ringo Ho, M. H., Colcombe, S. J., \& Kramer, A. F. (2005). A structural equation modeling analysis of attentional control: An event-related fMRI study. Brain Research. Cognitive Brain Research, 22, 349-357.

Fan, J., Flombaum, J. I., McCandliss, B. D., Thomas, K. M., \& Posner, M. I. (2003). Cognitive and brain consequences of conflict. Neuroimage, 18, 42-57. 
Fan, J., Hof, P. R., Guise, K. G., Fossella, J. A., \& Posner, M. I. (2008). The functional integration of the anterior cingulate cortex during conflict processing. Cerebral Cortex, 18, 796-805.

Fan, J., Kolster, R., Ghajar, J., Suh, M., Knight, R. T., Sarkar, R., et al. (2007). Response anticipation and response conflict: An event-related potential and functional magnetic resonance imaging study. Journal of Neuroscience, 27, 2272-2282.

Fassbender, C., Foxe, J. J., \& Garavan, H. (2006). Mapping the functional anatomy of task preparation: Priming task-appropriate brain networks. Human Brain Mapping, 27, 819-827.

Fellows, L. K., \& Farah, M. J. (2005). Is anterior cingulate cortex necessary for cognitive control? Brain, 128, 788-796.

Friston, K. J., Fletcher, P., Josephs, O., Holmes, A., Rugg, M. D., \& Turner, R. (1998). Event-related fMRI: Characterizing differential responses. Neuroimage, 7, 30-40.

Friston, K. J., Harrison, L., \& Penny, W. (2003). Dynamic causal modelling. Neuroimage, 19, 1273-1302.

Fuller, R., \& Jahanshahi, M. (1999). Impairment of willed actions and use of advance information for movement preparation in schizophrenia. Journal of Neurology, Neurosurgery and Psychiatry, 66, 502-509.

Garavan, H., Hester, R., Murphy, K., Fassbender, C., \& Kelly, C. (2006). Individual differences in the functional neuroanatomy of inhibitory control. Brain Research, 1105, 130-142.

Gehring, W. J., \& Knight, R. T. (2000). Prefrontal-cingulate interactions in action monitoring. Nature Neuroscience, 3, 516-520.

Green, J. J., \& McDonald, J. J. (2008). Electrical neuroimaging reveals timing of attentional control activity in human brain. PLoS Biology, 6, e81.

Grent-'t-Jong, T., \& Woldorff, M. G. (2007). Timing and sequence of brain activity in top-down control of visual-spatial attention. PLoS Biology, 5, e12.

He, B. J., Snyder, A. Z., Vincent, J. L., Epstein, A., Shulman, G. L., \& Corbetta, M. (2007). Breakdown of functional connectivity in frontoparietal networks underlies behavioral deficits in spatial neglect. Neuron, 53, 905-918.

Heim, S., Eickhoff, S. B., Ischebeck, A. K., Friederici, A. D., Stephan, K. E., \& Amunts, K. (2007). Effective connectivity of the left BA 44, BA 45, and inferior temporal gyrus during lexical and phonological decisions identified with DCM. Human Brain Mapping.

Hopfinger, J. B., Buonocore, M. H., \& Mangun, G. R. (2000). The neural mechanisms of top-down attentional control. Nature Neuroscience, 3, 284-291.

Huettel, S. A., Mack, P. B., \& McCarthy, G. (2002). Perceiving patterns in random series: Dynamic processing of sequence in prefrontal cortex. Nature Neuroscience, 5, 485-490.

Kastner, S., \& Ungerleider, L. G. (2000). Mechanisms of visual attention in the human cortex. Annual Review of Neuroscience, 23, 315-341.

Kerns, J. G., Cohen, J. D., MacDonald, A. W., III, Cho, R. Y., Stenger, V. A., \& Carter, C. S. (2004). Anterior cingulate conflict monitoring and adjustments in control. Science, 303, 1023-1026.

Kincade, J. M., Abrams, R. A., Astafiev, S. V., Shulman, G. L., \& Corbetta, M. (2005). An event-related functional magnetic resonance imaging study of voluntary and stimulus-driven orienting of attention. Journal of Neuroscience, 25, 4593-4604.

Knight, R. T. (2007). Neuroscience. Neural networks debunk phrenology. Science, 316, 1578-1579.

Knight, R. T., Grabowecky, M. F., \& Scabini, D. (1995). Role of human prefrontal cortex in attention control. Advances in Neurology, 66, 21-34; discussion 34-26.
Liang, H., Bressler, S. L., Ding, M., Truccolo, W. A., \& Nakamura, R. (2002). Synchronized activity in prefrontal cortex during anticipation of visuomotor processing. NeuroReport, 13, 2011-2015.

Liu, X., Banich, M. T., Jacobson, B. L., \& Tanabe, J. L. (2004) Common and distinct neural substrates of attentional control in an integrated Simon and spatial Stroop task as assessed by event-related fMRI. Neuroimage, 22, 1097-1106.

Liu, X., Banich, M. T., Jacobson, B. L., \& Tanabe, J. L. (2006) Functional dissociation of attentional selection within PFC: Response and non-response related aspects of attentional selection as ascertained by fMRI. Cerebral Cortex, 16, 827-834.

Luks, T. L., Simpson, G. V., Feiwell, R. J., \& Miller, W. L. (2002). Evidence for anterior cingulate cortex involvement in monitoring preparatory attentional set. Neuroimage 17, 792-802.

MacDonald, A. W., III, Cohen, J. D., Stenger, V. A., \& Carter, C. S. (2000). Dissociating the role of the dorsolateral prefrontal and anterior cingulate cortex in cognitive control. Science, 288, 1835-1838.

Margulies, D. S., Kelly, A. M., Uddin, L. Q., Biswal, B. B., Castellanos, F. X., \& Milham, M. P. (2007). Mapping the functional connectivity of anterior cingulate cortex Neuroimage, 37, 579-588.

Mechelli, A., Price, C. J., Noppeney, U., \& Friston, K. J. (2003). A dynamic causal modeling study on category effects: Bottom-up or top-down mediation? Journal of Cognitive Neuroscience, 15, 925-934.

Mesulam, M. M. (1981). A cortical network for directed attention and unilateral neglect. Annals of Neurology, 10, 309-325.

Mesulam, M. M. (1999). Spatial attention and neglect: Parietal, frontal and cingulate contributions to the mental representation and attentional targeting of salient extrapersonal events. Philosophical Transactions of the Royal Society of London, Series B, Biological Sciences, 354, 1325-1346.

Milham, M. P., Banich, M. T., Claus, E. D., \& Cohen, N. J. (2003). Practice-related effects demonstrate complementary roles of anterior cingulate and prefrontal cortices in attentional control. Neuroimage, 18, 483-493.

Miller, E. K. (2000). The prefrontal cortex and cognitive control. Nature Reviews Neuroscience, 1, 59-65.

Miller, E. K., \& Cohen, J. D. (2001). An integrative theory of prefrontal cortex function. Annual Review of Neuroscience, 24, 167-202.

Nee, D. E., Wager, T. D., \& Jonides, J. (2007). Interference resolution: Insights from a meta-analysis of neuroimaging tasks. Cognitive, Affective \& Behavioral Neuroscience, 7 , $1-17$.

Penny, W. D., Stephan, K. E., Mechelli, A., \& Friston, K. J. (2004). Comparing dynamic causal models. Neuroimage, 22, 1157-1172.

Pessoa, L., \& Ungerleider, L. G. (2004). Top-down mechanisms for working memory and attentional processes. In M. S. Gazzaniga (Ed.), The new cognitive neuroscience (3rd ed., pp. 919-930). Cambridge, MA: MIT Press.

Petit, L., Courtney, S. M., Ungerleider, L. G., \& Haxby, J. V. (1998). Sustained activity in the medial wall during working memory delays. Journal of Neuroscience, 18, 9429-9437.

Quintana, J., Wong, T., Ortiz-Portillo, E., Marder, S. R., \& Mazziotta, J. C. (2004). Anterior cingulate dysfunction during choice anticipation in schizophrenia. Psychiatry Research, 132, 117-130. 
Ridderinkhof, K. R., Ullsperger, M., Crone, E. A., \& Nieuwenhuis, S. (2004). The role of the medial frontal cortex in cognitive control. Science, 306, 443-447.

Rowe, J. B., Sakai, K., Lund, T. E., Ramsoy, T., Christensen, M. S., Baare, W. F., et al. (2007). Is the prefrontal cortex necessary for establishing cognitive sets? Journal of Neuroscience, 27, 13303-13310.

Rowe, J. B., Toni, I., Josephs, O., Frackowiak, R. S., \& Passingham, R. E. (2000). The prefrontal cortex: Response selection or maintenance within working memory? Science, 288, 1656-1660.

Slotnick, S. D., \& Schacter, D. L. (2004). A sensory signature that distinguishes true from false memories. Nature Neuroscience, 7, 664-672.

Stephan, K. E., Harrison, L. M., Kiebel, S. J., David, O., Penny, W. D., \& Friston, K. J. (2007). Dynamic causal models of neural system dynamics: Current state and future extensions. Journal of Biosciences, 32, 129-144.

Stephan, K. E., Marshall, J. C., Penny, W. D., Friston, K. J., \& Fink, G. R. (2007). Interhemispheric integration of visual processing during task-driven lateralization. Journal of Neuroscience, 27, 3512-3522.

Stephan, K. E., \& Penny, W. (2006). Dynamic causal models and Bayesian selection. In K. J. Friston (Ed.), Statistical parametric mapping: The analysis of functional brain images (pp. 577-585). Amsterdam: Elsevier.

Stephan, K. E., Penny, W. D., Marshall, J. C., Fink, G. R., \& Friston, K. J. (2005). Investigating the functional role of callosal connections with dynamic causal models. Annals of the New York Academy of Sciences, 1064 , 16-36.

Stephan, K. E., Weiskopf, N., Drysdale, P. M., Robinson, P. A., \& Friston, K. J. (2007). Comparing hemodynamic models with DCM. Neuroimage, 38, 387-401.

Strange, B. A., Duggins, A., Penny, W., Dolan, R. J., \& Friston, K. J. (2005). Information theory, novelty and hippocampal responses: Unpredicted or unpredictable? Neural Networks, 18, 225-230.

Sylvester, C. Y., Wager, T. D., Lacey, S. C., Hernandez, L., Nichols, T. E., Smith, E. E., et al. (2003). Switching attention and resolving interference: fMRI measures of executive functions. Neuropsychologia, 41, 357-370.

Wager, T. D., Jonides, J., \& Reading, S. (2004). Neuroimaging studies of shifting attention: A meta-analysis. Neuroimage, 22, 1679-1693.

Wager, T. D., \& Smith, E. E. (2003). Neuroimaging studies of working memory: A meta-analysis. Cognitive, Affective E Behavioral Neuroscience, 3, 255-274.

Wager, T. D., Sylvester, C. Y., Lacey, S. C., Nee, D. E., Franklin, M., \& Jonides, J. (2005). Common and unique components of response inhibition revealed by fMRI. Neuroimage, 27, 323-340.

Yago, E., Duarte, A., Wong, T., Barcelo, F., \& Knight, R. T. (2004). Temporal kinetics of prefrontal modulation of the extrastriate cortex during visual attention. Cognitive, Affective \& Behavioral Neuroscience, 4, 609-617. 